\title{
A Giant Solid-Cystic Mesenteric Fibromatosis with Unusual Synchronous Skin Fibromatosis
}

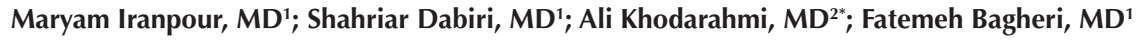 \\ ${ }^{1}$ Department of Pathology, Pathology and Stem Cell Research Center, Afzalipour Faculty of Medicine, Kerman University of Medical \\ Sciences, Kerman, Iran \\ ${ }^{2}$ Department of General Surgery, Pathology and Stem Cell Research Center, Afzalipour Faculty of Medicine, Kerman University of \\ Medical Sciences, Kerman, Iran
}

\begin{abstract}
Mesenteric fibromatosis (MF) is a rare, locally aggressive tumor without distant metastasis, which has a high recurrence rate. Based on its location, it is classified as intra-abdominal, from abdominal wall, and extra-abdominal. The incidence of cystic-solid, retroperitoneal tumors is very low in comparison to other MF forms. Intra-abdominal MFs are asymptomatic in early stages, but their symptoms appear late in the tumor course. There is no specific imaging finding since radiological diagnosis is mostly impossible. Thus, diagnosis is made histopathologically. Nowadays, there is no consensus about its treatment although surgical resection is widely used. In the present study, a very rare case of cystic-solid retroperitoneal MF associated with separate synchronous skin tumors is reported.

Keywords: Abdominal, Mesenteric fibromatosis, Tumor

Cite this article as: Iranpour M, Dabiri S, Khodarahmi A, Bagheri F. A giant solid-cystic mesenteric fibromatosis with unusual synchronous skin fibromatosis. Arch Iran Med. 2021;24(4):326-329. doi: 10.34172/aim.2021.45

Received: December 16, 2020, Accepted: January 10, 2021, ePublished: April 1, 2021
\end{abstract}

\section{Introduction}

Mesenteric fibromatosis (MF) is also known as mesenteric desmoid. 'Desmoid' is derived from the Greek word desmos meaning 'band of tendons'. ${ }^{1,2} \mathrm{MF}$ is a rare benign tumor composed of monoclonal proliferation of fibroblasts and myofibroblasts without any distant metastatic potential but with a high degree of local invasion and recurrence. These tumors mostly originated from the abdominal muscle layer and fascial sheath, ${ }^{3-5}$ and they usually occur sporadically, but familial adenomatous polyposis (FAP) and Gardner's Syndrome are the most common hereditary conditions associated with these tumors. ${ }^{6-8}$ Prior surgery, trauma, and high estrogen states can play an important role as predisposing factors. ${ }^{8}$ Approximately 5\%-10\% of the tumors occur in patients with FAP while 10\%-20\% of patients with these tumors develop FAP. Further, 16\% of the pediatric patients with this tumor have adenomatous polyposis coli (APC) mutations, while 85\%-90\% of the tumors include $\beta$-catenin mutations, having a significant correlation with an increased risk of postoperative recurrence. ${ }^{9}$ In addition, these tumors could be classified, based on their anatomical location, as intraabdominal, from the deep soft tissues of the abdominal wall, or deep within the extra-abdominal soft tissues. ${ }^{1}$ Only $5 \%$ of the sporadic tumors are intra-abdominal, but non-sporadic tumors mostly occur intraabdominally or on the abdominal wall. ${ }^{7}$ Retroperitoneal desmoid fibromatosis is rare and accounts for less than $1 \%$ of the retroperitoneal masses. ${ }^{10}$ Fibromatosis is asymptomatic in early stages. However, it is more likely for the tumor to become symptomatic as it grows, which could be mainly related to the compression effect of the tumor on nearby organs. Most cases are discovered when they are symptomatic while some are incidentally discovered when they are asymptomatic. Depending on its location and size, this tumor has a wide range of symptoms such as abdominal pain, nausea, vomiting, abdominal distention and loss of appetite, abdominal mass, gastrointestinal bleeding, small bowel obstruction, fistula formation, and bowel perforation, most of which are related to the small bowel. ${ }^{3,11,12}$ Lack of specific imaging features makes preoperative diagnosis extremely difficult. ${ }^{13}$ Surgical resection with a sufficient margin is the foundation of the treatment, but neither surgery nor chemotherapy/ radiotherapy constitute the principal therapy. Almost 50\% of the desmoid fibromatosis patients experience recurrence after surgery and/or radiotherapy. ${ }^{14}$ The present study aims to report a rare case of solid cystic retroperitoneal MF with multiple skin involvements.

\section{Case Report}

A 32-year-old man presented with progressive abdominal pain, nausea, vomiting, and weight loss since 6 weeks ago. On physical examination, he was reported as conscious,

*Corresponding Author: Ali Khodarahmi, MD; Department of General Surgery, Pathology and Stem Cell Research Center, Afzalipour Faculty of Medicine, Kerman University of Medical Sciences, Kerman, Iran. Tel: +98-34-33257112; Fax: +98-34-33257112; Email: khodarahmiali@yahoo.com 
cachectic, pale and suffering from nonspecific pain. The results of digital rectal exam was normal. His abdomen was boat-shaped without any distension. A non-tender, non-mobile, firm huge mass was palpated in the left half and right lower quadrant of his abdomen. No abdominal tenderness or guarding was detected. Further, there was no evidence of organomegaly or lymphadenopathy and the results of genital examination were normal. There were some firm, nontender skin lesions, similar to kelloid, especially on his trunk, which had gradually developed since he was 17 years old (Figure 1).

\section{Discussion}

A solid mass measuring $169^{*} 190^{*} 133 \mathrm{~mm}$ with a hypodense area inside was found by spiral multislice CT Scan near the left psoas muscle, the density of which was similar to muscle density. mass was enhanced by IV contrast and had a compression effect on the left ureter, causing severe left hydronephrosis. It appeared as a retroperitoneal mass. There was no organomegaly or lymphadenopathy (Figure 2). The results of genital ultrasonography was normal. Radilogical findings suggested a retroperitoneal sarcoma with necrosis inside the tumor.

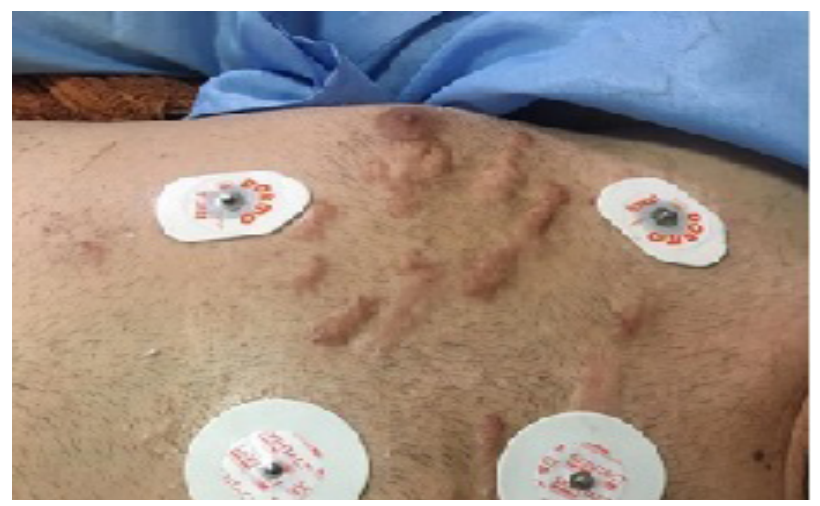

Figue 1. Kelloid-Shaped Skin Lesions on the Trunk
CT-guided transcutaneous core needle biopsy of the mass was performed. The specimen was processed for permenant histopathlogic evaluation. After $\mathrm{H} \&$ E staining, the mass looked like benign spindle cell tumors without any evidence of malignancy. Therefore, $\beta$-catenin staining was undertaken, and the pathology was compatible with fibromatosis.

During laparatomy, a huge asymmetrical, dumbbellshaped, and mass measuring $20 * 25 \mathrm{~cm}$ was found, the posterior part of which was smaller than its anterior part. In addition, it encased the middle part of the left ureter, the third part of duodenum, and a part of the superior mesentric artery. Some loops of jejunum were partially entrapped by the tumor. In addition, some loops of the small bowel had loose attachments to the mass. The mass was attached to the anterior abdominal wall by a loose and areolar type tissue. Resection of the tumor was carried out with a segment of the middle part of the left ureter, third portion of the duodenum, the superior mesentric artery, most of the jejuneum, and part of the anterior abdominal wall. Approximately, $90 \mathrm{~cm}$ of the terminal ileum and $60 \mathrm{~cm}$ of the jejuneum remained intact, and GI tract continuity was reestablished by jejunoduodenal and jejunoileal anastomosis as two seperate anastomoses. The end-end anastomosis of the left ureter over a stent was also performed. A second-look laparatomy was done, in which the ischemic ileocecal part of the bowel was resected and a jejunoduodenal anastomosis was performed.

Total parenteral nutrition was started on the second day after the last operation. After initating the oral diet, the TPN gradually changed to PPN, and finally stopped.

The patient was treated as a short bowel disease patient for approximately more than one year. Then, the condition was resolved, and he could completely tolerate the oral diet without any need for a supplementary diet, which led to the establishment of his weight at about $65 \mathrm{~kg}$ (body mass
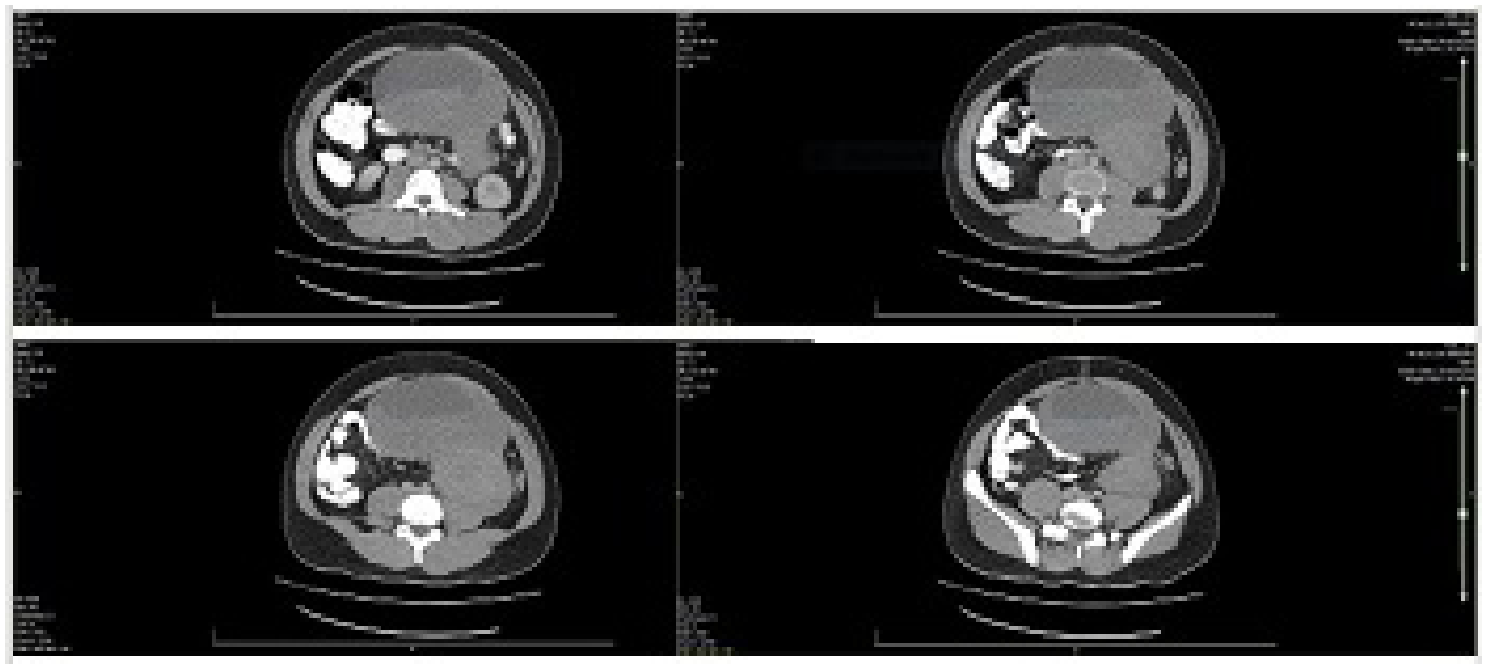

Figure 2. Spiral multislice CT scan imagings shows a solid-cystic, contrast-enhanced mass with a compression effect on the left ureter and left hydronephrosis. 
index $=23$ ).

Macroscopically, the specimen was a dumbbell-shaped, well-defined, partially capsulated, creamy brownish mass $(25 \times 25 \times 10 \mathrm{~cm})$, to which a piece of duodenal tissue $(2 \times 1$ $\mathrm{cm})$ was attached. The cut sections showed a creamy soft area and a cystic area $(9.5 \times 5 \mathrm{~cm})$ filled by a brownish fluid (Figure 3).

Histopathological studies revealed an exuberant fibrous matrix consisting of spindle cells with pale and eosinophilic cytoplasm, minimal mitotic activity, and without any atypia admixed kelloidal type collagenous bundles with foci of hemorrhage (Figure 4). The tumor infiltrated the serosal and muscular layer of the duodenum. Immunohistochemistry showed the tumor cells to be positive for $\beta$-catenin and vimentin, negative for S100, CD34, Desmin and CD117. In addition, Ki67 was positive in $2 \%$ of tumor cells (Figure 5 ).

He was followed up for forty-four months without any radiologic or physical evidence of recurrence. His only compliant was a vague abdominal pain, especially at his epigastrium.
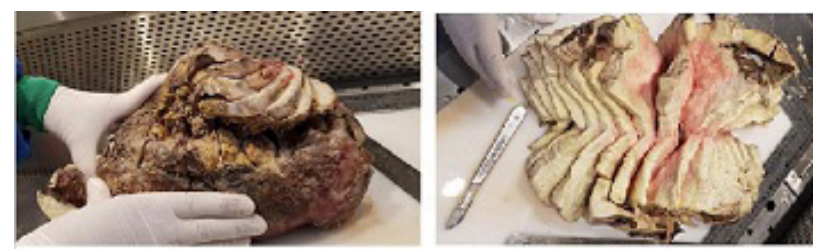

Figure 3. Gross Appearance of the Mass. Dumbbell-shaped, welldefined, partially capsulated, creamy brownish mass $(25 \times 25 \times 10$ $\mathrm{cm})$.

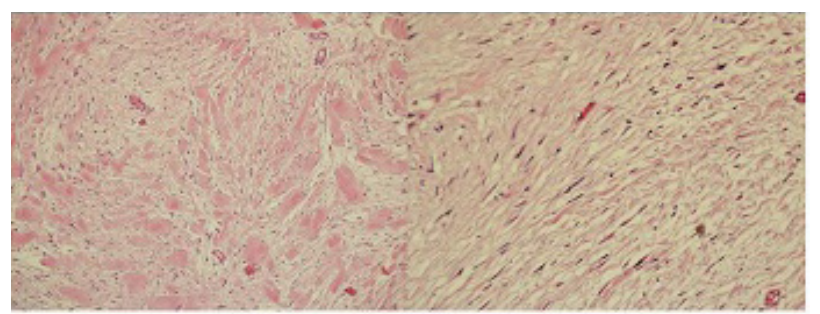

Figure 4. Spindled Cells of Uniform Appearance and Pale Cytoplasm Admixed Variably Sized Bands of Keloidal Collagen Fibers ( $\mathrm{H} \& \mathrm{E}$ Staining, $\times 100)$.

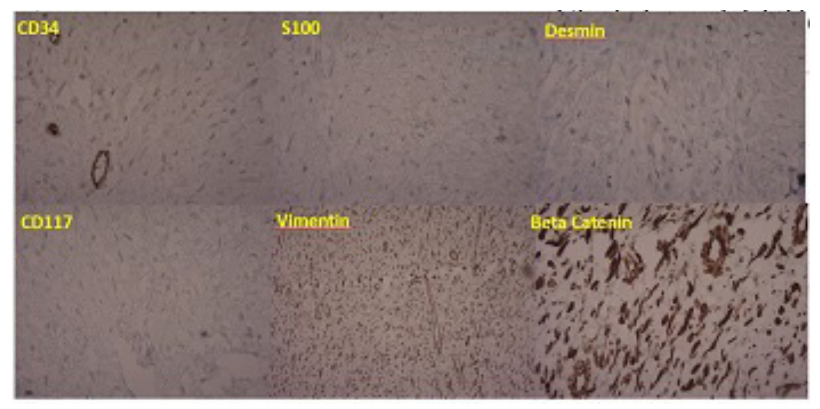

Figure 5. Immunohistochemistry Staining by CD34, S100, Desmin, CD117, Vimentin and Beta-Catenin Showing Negative Reaction for CD34, S100, Desmin, CD117 and Positive Reaction for Vimentin and Beta-Catenin $(\times 100)$.
MF is also known as mesenteric desmoid. 'Desmoid' is derived from the Greek word desmos meaning 'band of tendons'. ${ }^{1} \mathrm{MF}$ is a rare benign tumor composed of monoclonal proliferation of fibroblasts and myofibroblasts without any distant metastatic potential but with a high degree of local invasion and recurrence. These tumors mostly originate from the abdominal muscle layer and fascial sheath, ${ }^{2-4}$ and they usually occur sporadically, but FAP and Gardner's Syndrome are the most common hereditary conditions associated with these tumors. ${ }^{5,6}$ Approximately $5 \%-10 \%$ of the tumors occur in patients with FAP while $10 \%-20 \%$ of patients with these tumors develop FAP. Further, $16 \%$ of the pediatric patients with this tumor have APC mutations, while 85\%-90\% of the tumors include $\beta$-catenin mutations, having a significant correlation with an increased risk of postoperative recurrence. ${ }^{7}$ Lack of specific imaging features makes preoperative diagnosis extremely difficult. ${ }^{8}$ Surgical resection with a sufficient margin is the foundation of the treatment, but neither surgery nor chemotherapy/ radiotherapy constitute the principal therapy .Almost 50\% of the desmoid fibromatosis patients experience recurrence after surgery and/or radiotherapy. ${ }^{9}$ The present study aims to report a rare case of solid-cystic retroperitoneal MF with multiple skin involvements.

\section{Authors' Contribution}

MI and ShD particpated in pathology interpretation, image preparation and drafting the manuscript. Akh particpated in patient's surgical management and drafting the manuscript. FB contributed to image preparation and drafting the manuscript.

\section{Conflict of Interest Disclosures}

The authors have no conflicts of interest.

\section{Ethical Statement}

Informed consent was obtained from the patient's parents.

\section{Funding}

None.

\section{Acknowledgments}

The authors would like to thank professor Gunnlaugur Petur Nielsen for his support in diagnosing this case.

\section{References}

1. Gregory N, Tony K, Rasha S, Maroon T, Raja W. Incidental Finding and Management of Mesenteric Fibromatosis. Am J Case Rep. 2016;17(1):389-94. doi: 10.12659/AJCR.898122.

2. Jiannan L, Hanxiang L, Wei C, Yan Z, Lifang J, Tongjun L, et al. Duodenum-derived fibromatosis that invaded the muscular layer of intestinal wall: A rare case report. Medicine. 2017;96(31):e7684. doi: 10.1097/MD.0000000000007684.

3. Wang J, Huang Y, Sun Y, Ge Y, Zhang M. Value of imaging findings in predicting post-operative recurrence of desmoidtype fibromatosis. Oncol Lett. 2020;19(1):869-75. doi: 


\subsection{2/ol.2019.11129}

4. Yoshitoshi I, Tamaki M, Gaku M, Satoshi I, Takaomi H, Kimiaki $\mathrm{H}$, et al. A mesenteric solid tumor with unusual features in a young male: a case report. Mol Clin Oncol. 2017;7(3):355-8. doi: 10.3892/mco.2017.1344.

5. Wang Y, Jiang B, Yang Y, Wang S, Li Y, Meng N, et al. Gastric aggressive fibromatosis: report of a case and review of the literature. Int J Clin Exp Pathol. 2019;12(1):372-7.

6. Palladino E, Nsenda J, Siboni R, Lechner C. A giant mesenteric desmoid tumor revealed by acute pulmonary embolism due to compression of the inferior vena cava. Am J Case Rep. 2014;2(15):374-7. doi: 10.12659/AJCR.891044.
7. Hapgood C, DeLong A. Recurrent Enlarging Mesenteric DesmoidTumor following Remote Surgical Resection. Case Rep Radiol. 2017;2017:2312617. doi: 10.1155/2017/2312617.

8. Abhinav M, Mohinder S, Anoop V, Gunjeet S, Malwinder S, Rupesh N. Mesenteric Fibromatosis Presenting as a Diagnostic Dilemma: A Rare Differential Diagnosis of Right Iliac Fossa Mass in an Eleven Year Old-A Rare Case Report. Case Rep Surg. 2013;2013:569578. doi: 10.1155/2013/569578.

9. Omi M, Kanao H, Aoki $\mathrm{Y}$, Okamoto $\mathrm{S}$, Takeshima N. Minimally invasive diagnostic and therapeutic surgery for an intraabdominal desmoid tumor: A case report. Gynecol Oncol Rep. 2020;32:100560. doi: 10.1016/j.gore.2020.100560. 